\title{
GUIDED WAVE PROPAGATION IN THERMAL MEDIA THROUGH THE SEMI ANALYTICAL FINITE ELEMENT METHOD
}

\author{
Faker Bouchoucha, Sonda ChaAbane \\ National School of Engineers of Sfax, Unit of Dynamics of the Mechanical Systems, Sfax, Tunisia \\ e-mail: fakersbouchoucha@yahoo.fr; sonda.chaabene@ec-lyon.fr \\ Mohamed NaJib ICHCHOU \\ Ecole Centrale de Lyon, Laboratory of Tribology and Dynamics of Systems (LTDS), Lyon, France \\ e-mail: mohamed.ichchou@ec-lyon.fr \\ MOHAMED HADDAR \\ National School of Engineers of Sfax, Unit of Dynamics of the Mechanical Systems, Sfax, Tunisia \\ e-mail: mohamed.haddar@enis.rnu.tn
}

\begin{abstract}
In this paper, the issue of the estimation of wave propagation characteristics in thermal media is dealt with. A formulation, named the Thermal Semi Analytical Finite Element, based on the semi analytical finite element approach coupled with the thermal effect is offered. Temperature variations affect the mechanical properties of the waveguide. The question of dispersion curves and group velocities is studied. This study is expected to be of use in the sensitivity analysis of guided waves for wave propagation in thermal environment. Comparisons between numerical and analytical results are given to show the effectiveness of the proposed approach.
\end{abstract}

Keywords: semi analytical finite element, thermal media, dispersion, velocity

\section{Introduction}

Guided waves are still a subject of intensive research in several engineering areas. This research focuses on the study of guided wave properties and applications. Structural Health Monitoring (SHM) and Non Destructive Testing (NDT) are among the fields of application of this numerical tool. To study wave propagation in structural waveguides, the semi analytical finite element (SAFE) method has been investigated by many researchers. Hayashi et al. (2003) derived the SAFE formulation through virtual work principles and proposed a way to calculate the group velocity using the eigensolution at a given frequency. Damljanovic and Weaver (2004) developed linear triangular elements for the SAFE method using Lagrange's equations to investigate elastic waves in waveguides of arbitrary cross-section. Gavric (1995) calculated the dispersion relationship in a free rail by using triangular and quadrilateral elements obtained from Hamilton's principle. The SAFE method was also adopted to investigate wave propagation characteristics for thin-walled structures by Finnveden (2004), where the polynomial interpolation was used in the propagation axis thus leading to polynomial eigenvalue problems. The method was extended to curved structures by Finnveden and Fraggstead (2008), where isoparametric elements were used.

In the current work, the semi analytical numerical method that may be used for wave propagation and dynamic analysis of waveguide structures is presented. The basic formulations are investigated to illustrate the merits and shortcomings of the method through the virtual work principle. 
The effect of temperature on guided wave structural health monitoring has been studied by several authors in the literature, see e.g. Konstantinidis et al. (2006). They attempted to correlate modal properties with temperature and also to develop system identification models that could separate the influences of temperature from true indications of damage on dynamic modal parameters (Alashti and Kashiri, 2010). Their research effort extensively examined the causes of the effects of temperature and how they affect dynamic characteristics in a normal real-life of a beam. Some authors have been interested in studying the impact of thermal loading on the guided waves mode shape. A useful research was reported on isotropic beams, plates and shells. Jeyaraj et al. (2009) studied the vibration and acoustic response of a composite plate in thermal environment. Kadoli and Ganesan (2006) studied the dynamic behavior of composite and isotropic cylindrical shells with PZT layers under axisymmetric temperature variation.

In this work, the effect of temperature on the wave propagation is studied through the proposed approach, named the thermal semi analytical finite element (TSAFE) method. Indeed, we combine the semi analytical finite element method with thermal treatment to show the effect of temperature on the characteristics of guided wave propagation.

Temperature variations affect various mechanical properties of the structure such as elasticity modulus, density, etc. The modelling of the structure under thermal environment is still a subject of intensive research in several engineering areas. Experimental and theoretical results are offered in many researches to study the material behaviour following thermal variability and evaluate the high-temperature thermal and mechanical properties of the material (Kodur et al., 2012; Li et al., 2013).

The origin of this work is the treatment of wave characteristics (dispersion curves and group velocity) as a function of temperature in order to study the thermal effect on the semi analytical finite element method together with analytical and numerical validations.

\section{Description of the Thermal Semi Analytical Finite Element (TSAFE) method}

In this Section, we introduce the TSAFE method that may be used for wave propagation and dynamic analysis of waveguide structures in the presence of the thermal effect. Consider a structural waveguide with a uniform cross section. Under thermal environment, the weak form based on the virtual work principle for the dynamic problem may be written as (Hayashi et al., 2003; Gavric 1995)

$$
W^{e}\left(u^{*}, u, T\right)=\int_{V}\left\langle\varepsilon_{d e f}^{*}(T)\right\rangle \boldsymbol{\sigma}(T) d v+\int_{V}\left\langle\mathbf{u}^{*}\right\rangle \rho(T) \ddot{\mathbf{u}} d v=W_{\text {int }}(T)-W_{\text {ext }}(T)=0
$$

where $W^{e}$ is the thermal virtual work of the internal forces, $T$ is temperature, $\rho(T)$ is density at temperature $T,{ }^{*}$ denotes virtual quantities, $\varepsilon_{d e f}(T)=\left[\varepsilon_{x x}(T), \varepsilon_{y y}(T), \varepsilon_{z z}\right.$, $\left.2 \varepsilon_{x y}(T), 2 \varepsilon_{y z}(T), 2 \varepsilon_{x z}(T)\right]^{\mathrm{T}}$ is the strain vector at temperature $T, \mathbf{u}=\left[u_{x}, u_{y}, u_{z}\right]^{\mathrm{T}}$ is the displacement field, $\boldsymbol{\sigma}(T)=\left[\sigma_{x x}(T), \sigma_{y y}(T), \sigma_{z z}(T), \sigma_{x y}(T), \sigma_{y z}(T), \sigma_{x z}(T)\right]^{\mathrm{T}}$ is the stress vector at temperature $T$. $W_{\text {int }}(T)=\int_{V}\left\langle\varepsilon_{d e f}^{*}(T)\right\rangle \boldsymbol{\sigma}(T) d v$ and $W_{\text {ext }}(T)=-\int_{V}\left\langle\mathbf{u}^{*}\right\rangle \rho(T) \ddot{\mathbf{u}} d v$ are the internal and external virtual work at temperature $T$, respectively. The harmonic waves in a uniformly cross-sectioned waveguide are described by the orthogonal function $\exp (j \omega t-j k x)$, where $k$ is the wave number in the $x$ direction, $\omega$ is the circular frequency. The displacement function can be $u(x, y, z, t)=u(y, z) \exp (j \omega t-j k x)$, where $u(y, z)$ describes the amplitudes of the displacements of the waveguide cross-section. Thus the strain-displacement relationship $\varepsilon_{\text {def }}(T)=\mathbf{D}(T) \mathbf{u}$, where $\mathbf{D}(T)$ is a differential operator in the presence of the thermal effect, becomes

$$
\varepsilon_{d e f}(T)=\mathbf{D}_{0}(T)+k \mathbf{D}_{1} \mathbf{u}
$$


Similar to the standard FE method, the natural coordinates can be employed to facilitate the use of the standard Gauss integration formulas. The same shape functions are employed to specify the relation between the global $(x, y)$ and local $(\varsigma, \eta)$ coordinate systems. By inserting the interpolation of the displacement functions in the strain-displacement relationship, we obtain

$$
\varepsilon_{d e f}(T)=\mathbf{D}(T) \mathbf{N u}_{i}
$$

where $\mathbf{u}_{i}=\left[u_{x_{i}}, u_{y_{i}}, u_{z_{i}}\right] 6 \mathrm{~T}$ is the displacement vector of the finite element, $\mathbf{N}$ is the matrix of the element shape functions.

The relationship between the strain and the stress vectors can be given in the following manner

$$
\boldsymbol{\sigma}(T)=\mathbf{C}(T) \boldsymbol{\varepsilon}_{d e f}(T)
$$

where $\mathbf{C}(T)$ is the material stiffness matrix at temperature $T$.

The external virtual work at temperature $T$ can be developed as follows

$$
W_{\text {ext }}(T)=\left\langle\mathbf{u}_{i}^{*}\right\rangle \mathbf{M}^{e}(T) \ddot{\mathbf{u}}_{i}
$$

where $\mathbf{M}^{e}(T)$ is the mass matrix at temperature $T$, which can be given as

$$
\mathbf{M}^{e}(T)=\int_{\Omega^{e}} \rho(T) \mathbf{N}^{\mathrm{T}} \mathbf{N} d \Omega^{e}
$$

where $\Omega^{e}$ denotes the element domain.

The internal thermal virtual work can be developed as

$$
W_{\text {int }}(T)=\left\langle\mathbf{q}_{i}^{*}\right\rangle \mathbf{K}^{e}(T) \mathbf{q}_{i}
$$

where $\mathbf{K}^{e}(T)$ is the stiffness matrix at temperature $T$, which can be written as

$$
\mathbf{K}^{e}(T)=\int_{\Omega^{e}} \mathbf{N}^{\mathrm{T}}[\mathbf{D}(T)]^{\mathrm{T}} \mathbf{C}(T) \mathbf{D}(T) \mathbf{N} d \Omega^{e}
$$

Introducing equation (2.2) into equation (2.8) leads to

$$
\mathbf{K}^{e}(T)=\int_{\Omega^{e}} \mathbf{N}^{\mathrm{T}}\left[\mathbf{D}_{0}(T)+k \mathbf{D}_{1}\right]^{\mathrm{T}} \mathbf{C}(T)\left[\mathbf{D}_{0}(T)+k \mathbf{D}_{1}\right] \mathbf{N} d \Omega^{e}
$$

Then

$$
\mathbf{K}^{e}(T)=\mathbf{K}_{0}^{e}(T)+k \mathbf{K}_{1}^{e}(T)+k^{2} \mathbf{K}_{2}^{e}(T)
$$

where

$$
\begin{aligned}
& \mathbf{K}_{0}^{e}(T)=\int_{\Omega^{e}} \mathbf{N}^{\mathrm{T}} \mathbf{D}_{0}^{\mathrm{T}}(T) \mathbf{C}(T) \mathbf{D}_{0}(T) \mathbf{N} d \Omega^{e} \\
& \mathbf{K}_{1}^{e}(T)=\int_{\Omega^{e}} \mathbf{N}^{\mathrm{T}} \mathbf{D}_{1}^{\mathrm{T}} \mathbf{C}(T) \mathbf{D}_{0}(T) \mathbf{N} d \Omega^{e}+\int_{\Omega^{e}} \mathbf{N}^{\mathrm{T}} \mathbf{D}_{0}^{\mathrm{T}}(T) \mathbf{C}(T) \mathbf{D}_{1} \mathbf{N} d \Omega^{e} \\
& \mathbf{K}_{2}^{e}(T)=\int_{\Omega^{e}} \mathbf{N}^{\mathrm{T}} \mathbf{D}_{1}^{\mathrm{T}} \mathbf{C}(T) \mathbf{D}_{1} \mathbf{N} d \Omega^{e}
\end{aligned}
$$


The assembly of the element matrices and vectors leads to the governing equation of motion of the waveguide in the presence of the thermal effect

$$
\left[\mathbf{K}_{0}(T)-\omega^{2} \mathbf{M}(T)+k \mathbf{K}_{1}(T)+k^{2} \mathbf{K}_{2}(T)\right] \boldsymbol{\varphi}=\mathbf{0}
$$

where $\varphi$ denotes the nodal displacement vector.

Suppose $\varphi_{1}$ and $\varphi_{2}$ denote the eigenvectors associated to $k$ and $-k$, respectively. From the equation of motion, we have

$$
\begin{aligned}
& {\left[\mathbf{K}_{0}(T)-\omega^{2} \mathbf{M}(T)+k \mathbf{K}_{1}(T)+k^{2} \mathbf{K}_{2}(T)\right] \boldsymbol{\varphi}_{1}=\mathbf{0}} \\
& {\left[\mathbf{K}_{0}(T)-\omega^{2} \mathbf{M}(T)-k \mathbf{K}_{1}(T)+k^{2} \mathbf{K}_{2}(T)\right] \boldsymbol{\varphi}_{2}=\mathbf{0}}
\end{aligned}
$$

The linearization of the equation of motion can be given in the following form

$$
\left[\begin{array}{cc}
\mathbf{K}_{1}(T) & \mathbf{K}_{0}(T)-\omega^{2} \mathbf{M}(T) \\
\mathbf{K}_{0}(T)-\omega^{2} \mathbf{M}(T) & 0
\end{array}\right]\left\{\begin{array}{c}
k \boldsymbol{\phi}_{1} \\
\boldsymbol{\phi}_{2}
\end{array}\right\}+k^{2}\left[\begin{array}{cc}
0 & \mathbf{K}_{2}(T) \\
\mathbf{K}_{2}(T) & \mathbf{K}_{1}(T)
\end{array}\right]\left\{\begin{array}{c}
k \boldsymbol{\phi}_{1} \\
\boldsymbol{\phi}_{2}
\end{array}\right\}=\mathbf{0}
$$

where $\phi_{1}=\varphi_{1}+\varphi_{2}$ and $\phi_{2}=\varphi_{1}-\varphi_{2}$.

The linearized equation of motion presents the eigenvalue problem of the system

$$
[\mathbf{A}(T)-\lambda \mathbf{B}(T)] \phi=\mathbf{0}
$$

where $\boldsymbol{\phi}=\left[k \phi_{1}, \phi_{2}\right]^{\mathrm{T}}, \lambda=k^{2}$ and

$$
\mathbf{A}(T)=\left[\begin{array}{cc}
\mathbf{K}_{1}(T) & \mathbf{K}_{0}(T)-\omega^{2} \mathbf{M}(T) \\
\mathbf{K}_{0}(T)-\omega^{2} \mathbf{M}(T) & 0
\end{array}\right] \quad \mathbf{B}(T)=-\left[\begin{array}{cc}
0 & \mathbf{K}_{2}(T) \\
\mathbf{K}_{2}(T) & \mathbf{K}_{1}(T)
\end{array}\right]
$$

The resolution of this eigenvalue problem leads to calculation of thermal characteristics of the travelling waves.

It can be of interest, in many applicative engineering cases, to consider the wave numbers and velocities and to provide the dispersion curves. Indeed, from the knowledge of the eigenvalue, the wave numbers at temperature $T$ can be extracted as follows

$$
k(T)= \pm \sqrt{\lambda(T)}
$$

And the group velocities can be written as

$$
C g(T)=\frac{\partial \omega}{\partial k(T)}
$$

\section{Numerical results and discussion}

In this Section, numerical results are presented and discussed in order to study the efficiency of the proposed method as a tool for guided wave propagation under thermal environment. The numerical simulations are treated using the software MATLAB.

\subsection{Validation of the TSAFE method}

In this Section, we study the case of a longitudinal wave in order to validate the TSAFE formulation by comparisons with the analytical results. The waveguide is assimilated to the beam element with 2 nodes and 1 dof per node. The used material is steel $\left(\rho=7800 \mathrm{~kg} / \mathrm{m}^{3}\right.$, $\left.\nu=0,3, E=2 \cdot 10^{11} \mathrm{~Pa}\right)$. 
The thermal mass and stiffness matrices for the traction compression mode are

$$
\mathbf{M}^{\text {tract-comp }}=\frac{\rho S d}{6}\left[\begin{array}{ll}
2 & 1 \\
1 & 2
\end{array}\right] \quad \mathbf{K}^{\text {tract-comp }}=\frac{E S}{d}\left[\begin{array}{cc}
1 & -1 \\
-1 & 1
\end{array}\right]
$$

where $E$ is Young's modulus, $S$ is cross section area, $\rho$ is mass density and $d$ is length of the considered element.

Experimental and theoretical results are offered in many researches works on the behaviour of mechanical properties in the presence of thermal environment. The variation of Young's modulus of steel in the temperature range $\left[25^{\text {circ }} \mathrm{C}-1000^{\text {circ }} \mathrm{C}\right]$ can be estimated as follows (French Standard, 2007)

$$
\frac{E(T)}{E}=1+\frac{T}{2000 \log \frac{T}{1100}}
$$

where $E(T)$ is Young's modulus at temperature $T$.

The thermal elongation of steel can be governed through the following equation

$$
\frac{d(T)}{d}=1+\alpha \Delta T
$$

where $\alpha$ is the linear thermal expansion coefficient $\left(\alpha_{\text {steel }}=1.27 \cdot 10^{-5} \mathrm{~K}^{-1}\right), d(T)$ is length of the structure at temperature $T, \Delta T$ is the temperature variation.

The effect of the thermal gradient on density can be given as follows

$$
\frac{\rho(T)}{\rho}=\frac{1}{1+\gamma \Delta T}
$$

where $\gamma=3 \alpha$ is the volumetric thermal expansion and $\rho(T)$ is the density at temperature $T$.

In the presence of the thermal effect, the mass and stiffness matrices, for the longitudinal mode, can be given as follows

$$
\begin{aligned}
& \mathbf{M}^{\text {tract-comp }}(T)=\frac{\rho(T) S d(T)}{6}\left[\begin{array}{ll}
2 & 1 \\
1 & 2
\end{array}\right]=\frac{(1+\alpha \Delta T)}{(1+\gamma \Delta T)} \frac{\rho S d}{6}\left[\begin{array}{ll}
2 & 1 \\
1 & 2
\end{array}\right] \\
& \mathbf{K}_{0}^{\text {tract-comp }}(T)=\frac{E(T) S}{d(T)}\left[\begin{array}{cc}
1 & -1 \\
-1 & 1
\end{array}\right]=\frac{T+2000 \log \frac{T}{1100}}{2000(1+\alpha \Delta T) \log \frac{T}{1100}} \frac{E S}{d}\left[\begin{array}{cc}
1 & -1 \\
-1 & 1
\end{array}\right] \\
& \mathbf{K}_{1}^{\text {tract-comp }}=\left(1+\frac{T}{2000 \log \frac{T}{1100}}\right) \frac{1}{1+\alpha \Delta T} j \frac{E S}{2 d}\left[\begin{array}{cc}
1 & 0 \\
0 & -1
\end{array}\right] \\
& \mathbf{K}_{2}^{\text {tract-comp }}=-\left(1+\frac{T}{2000 \log \frac{T}{1100}}\right) \frac{E S}{3 d}\left[\begin{array}{cc}
1 & 1 / 2 \\
1 / 2 & 1
\end{array}\right]
\end{aligned}
$$

The numerical accuracy and the computational efficiency of the TSAFE method can be demonstrated by comparison with the analytical results given, for the longitudinal mode, in the following form

$$
k_{\text {analytical tract-comp }}(T)=\omega \sqrt{\frac{\rho(T)}{E(T)}}=\omega \sqrt{\frac{\rho}{E}} \sqrt{\frac{2000 \log \frac{T}{1100}}{(1+\gamma \Delta T)\left(T+2000 \log \frac{T}{1100}\right)}}
$$

Figure 1 illustrates variation of the wave number according to temperature for the longitudinal mode. The thermal effect is treated at different frequencies to study the influence of the coupling phenomenon between the temperature and frequency. The temperature elevation causes a small 


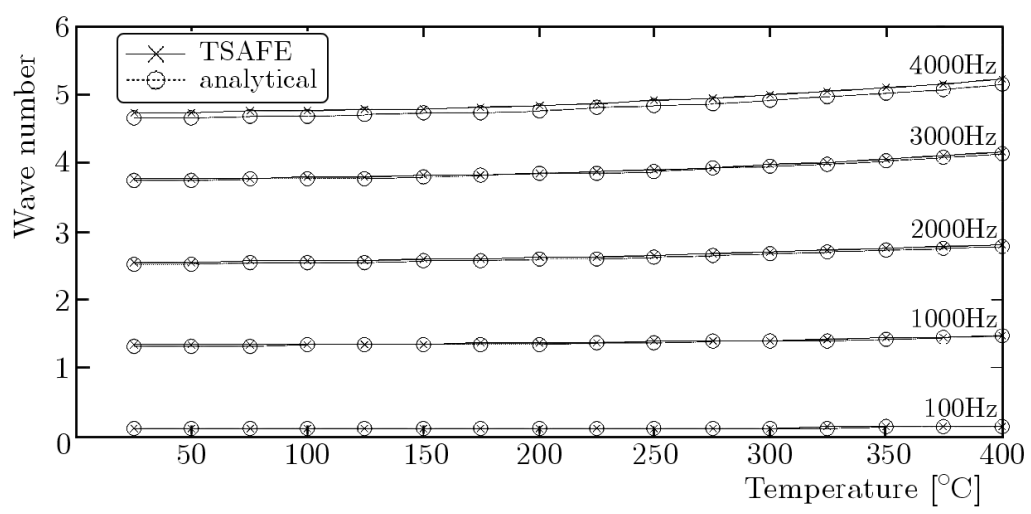

Fig. 1. Thermal dispersion curves for the longitudinal mode

increase in the dispersion curves. This increase is cleared at a high frequency. A good concordance is shown between the TSAFE and analytical results in the domain of the study.

The group velocity is generally used to study the dispersive behavior of the traveling mode. Figure 2 shows the evolution of the group velocity for the traction compression mode according to temperature at $f=3500 \mathrm{~Hz}$. The wave velocity decreases with temperature.

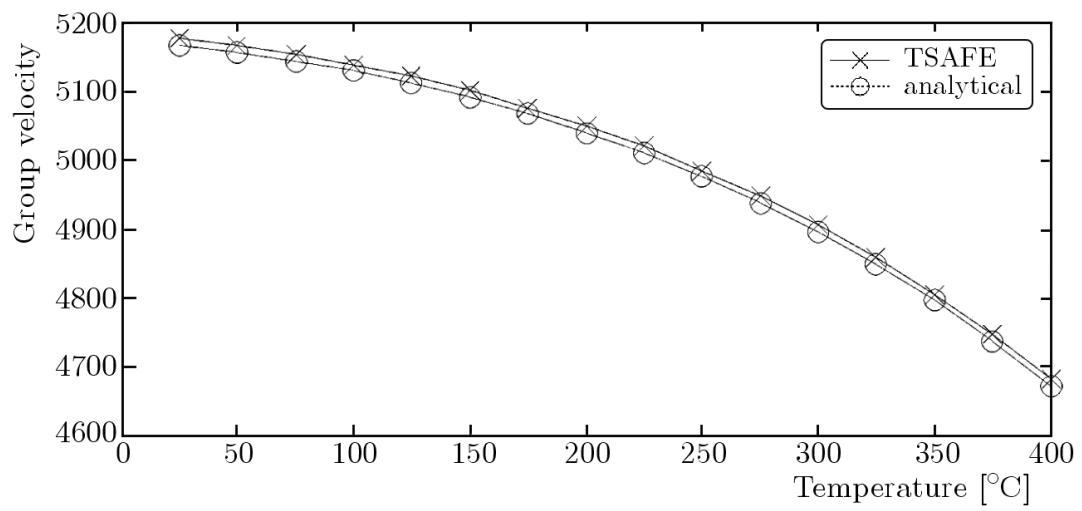

Fig. 2. Thermal group velocity for the longitudinal mode $(f=3500 \mathrm{~Hz})$

\subsection{Multimodal propagation through the TSAFE method}

In this Section, the TSAFE method is generalized for multimodal propagation to study the temperature effect on the traveling modes such as longitudinal, torsional, flexural and cross sectional modes. The simulation of the dispersion curves and the group velocities of wave propagation in a cylindrical pipe under thermal environment are the objective of this Subsection.

The TSAFE method is applied through the cylindrical pipe (Fig. 3). The used material is steel. We use a surface element with 4 nodes that include 2 dof per node.

The material stiffness matrix at temperature $T$ can be written using the cylindrical coordinate space as

$$
\mathbf{C}(T)=\frac{E(T)}{(1+\nu)(1-2 \nu)}\left[\begin{array}{ccc}
1-\nu & \nu & 0 \\
\nu & 1-\nu & 0 \\
0 & 0 & (1-2 \nu) / 2
\end{array}\right]
$$

The displacement field through the cylindrical coordinate is

$$
u(r, \theta, z, t)=u(r, \theta) \exp [j(\omega t-k z)]
$$




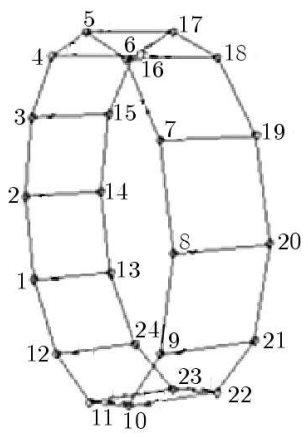

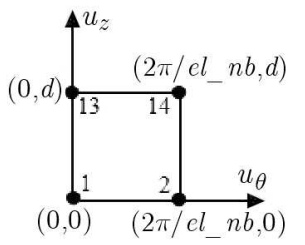

Global coordinate systems

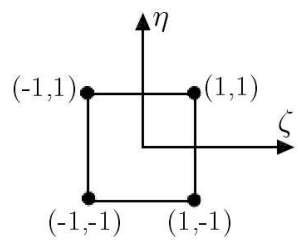

Local coordinate systems

Fig. 3. Substructure of cylindrical pipe

The strain-displacement relationship can be written in the following form

$$
\varepsilon_{d e f}=\mathbf{D u}(r, \theta, z, t)
$$

In the presence of thermal environment, the differential operator $\mathbf{D}(T)$ at temperature $T$ is written as

$$
\begin{aligned}
\mathbf{D}(T) & =\left[\begin{array}{cc}
\frac{1}{r(T)} \frac{\partial}{\partial \theta} & 0 \\
0 & \frac{\partial}{\partial z} \\
\frac{\partial}{\partial z} & \frac{1}{r(T)} \frac{\partial}{\partial \theta}
\end{array}\right]=\frac{1}{1+\alpha \Delta T}\left[\begin{array}{cc}
\frac{1}{r} \frac{\partial}{\partial \theta} & 0 \\
0 & 0 \\
0 & \frac{1}{r} \frac{\partial}{\partial \theta}
\end{array}\right]+k\left[\begin{array}{cc}
0 & 0 \\
0 & -j \\
-j & 0
\end{array}\right] \\
& =\frac{1}{1+\alpha \Delta T} \mathbf{D}_{0}+k \mathbf{D}_{1}
\end{aligned}
$$

The stiffness matrices at temperature $T$ can be given in the following equations

$$
\begin{aligned}
& \mathbf{K}_{0}^{e}(T)=\left(1+\frac{T}{2000 \log \frac{T}{1100}}\right) \frac{1}{(1+\alpha \Delta T)^{2}} \int_{\Omega^{e}} \mathbf{N}^{\mathrm{T}} \mathbf{D}_{0}^{\mathrm{T}} \mathbf{C} \mathbf{D}_{0} \mathbf{N} d \Omega^{e} \\
& \mathbf{K}_{1}^{e}(T)=\left(1+\frac{T}{2000 \log \frac{T}{1100}}\right) \frac{1}{1+\alpha \Delta T}\left(\int_{\Omega^{e}} \mathbf{N}^{\mathrm{T}} \mathbf{D}_{1}^{\mathrm{T}} \mathbf{C} \mathbf{D}_{0} \mathbf{N} d \Omega^{e}+\int_{\Omega^{e}} \mathbf{N}^{\mathrm{T}} \mathbf{D}_{0}^{\mathrm{T}} \mathbf{C} \mathbf{D}_{1} \mathbf{N} d \Omega^{e}\right) \\
& \mathbf{K}_{2}^{e}(T)=\left(1+\frac{T}{2000 \log \frac{T}{1100}}\right) \int_{\Omega^{e}} \mathbf{N}^{\mathrm{T}} \mathbf{D}_{1}^{\mathrm{T}} \mathbf{C} \mathbf{D}_{1} \mathbf{N} d \Omega^{e}
\end{aligned}
$$

Using equation (3.4), the mass matrix at temperature $T$ can be given as follows

$$
\mathbf{M}^{e}(T)=\frac{1}{1+\gamma \Delta T} \int_{\Omega^{e}} \rho \mathbf{N}^{\mathrm{T}} \mathbf{N} d \Omega^{e}
$$

In Fig. 4, the dispersion curves for multimodal propagation are presented according to temperature at $f=4000 \mathrm{~Hz}$. We can note the thermal effect on the wave number, in particular on the cross sectional mode. Generally, we can confirm that the wave number increases with temperature for the multimodal propagation.

Figure 5 presents the evolution of group velocity for multimodal propagation according to temperature at $f=4000 \mathrm{~Hz}$. The wave velocity decreases with temperature. We can say that the dispersive behavior of the traveling modes is affected by temperature elevation in the structure. 


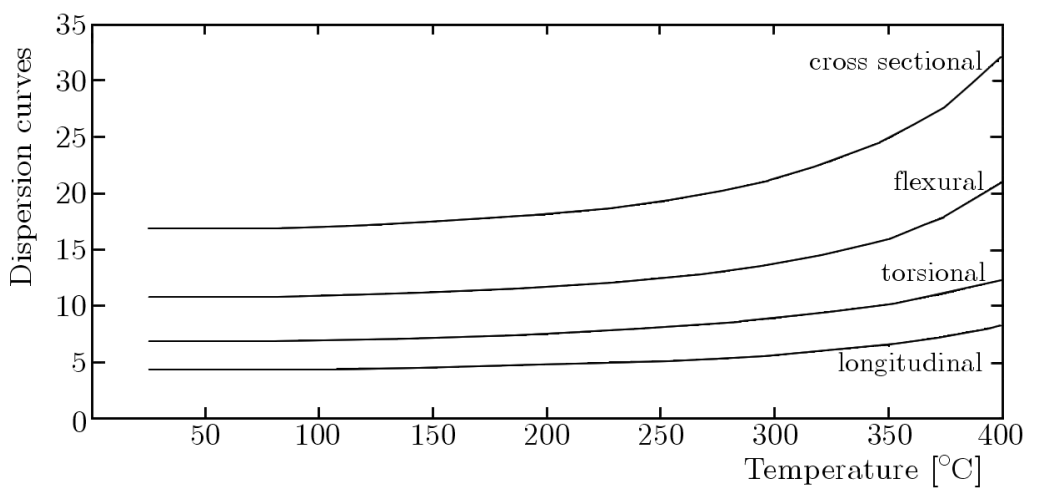

Fig. 4. Thermal dispersion curves for multimodal propagation by the TSAFE method $(f=4000 \mathrm{~Hz})$

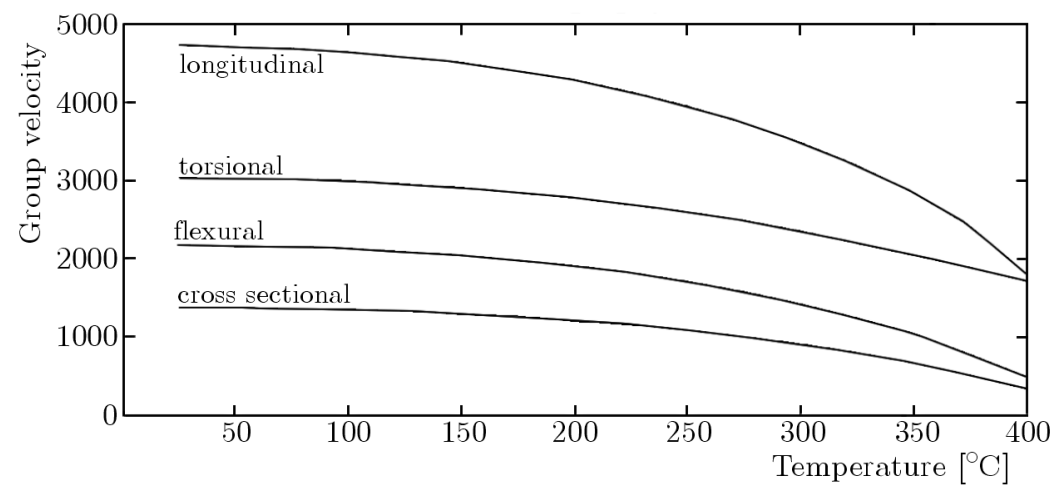

Fig. 5. Thermal group velocity for multimodal propagation by the TSAFE method $(f=4000 \mathrm{~Hz})$

In conclusion, this study shows the temperature effect on the characteristics of the guided waves such as dispersion and velocity. The temperature elevation causes augmentation of the dispersion curves and attenuation of the group velocity. But the guided waves save their efficiency to propagate through the structure at $T \leqslant 400^{\circ} \mathrm{C}$ and $f \leqslant 4000 \mathrm{~Hz}$.

\section{Conclusion}

In this paper, the issue of wave propagation parameters estimation in thermal environment through the TSAFE method is dealt with. The proposed approach allows wave characteristics to be defined by dispersion curves and group velocities through thermal media. The thermal effect is introduced into the structural parameters, and by making use of the finite element techniques, the behavior of the wave dispersion is studied. Ultimately, analytical comparisons are given. The main paper findings can be extracted as follows:

- The TSAFE method based on the virtual work principle in the presence of the thermal effect is developed.

- The guided wave propagation characteristics defined by dispersion curves and group velocities are studied under thermal environment.

- The numerical accuracy and the computational efficiency of this method are demonstrated by comparison with the analytical results.

The TSAFE offers some interesting research perspectives. The use of TSAFE for energy issues in a complex wave guide is an important task in our future work. Further investigations are under progress in order to use such numerical methods in the context of smart materials and 
structures. In addition of the mentioned axis, the proposed numerical method will be extended soon to the control of wave propagation in two-dimensional structures.

\section{References}

1. Alashti R.A., Kashini N., 2010, The effect of temperature variation of a simply supported curved sandwich beam with a flexible core, Mechanical Engineering Science, 225, 537-547

2. Damljanovic V., Weaver R.L., 2004, Propagating and evanescent elastic waves in cylindrical waveguides of arbitrary cross section, Journal of the Acoustical Society of America, 115, 1572-1581

3. Finnveden S., 2004, Evaluation of modal density and group velocity by a finite element method, Journal of Sound and Vibration, 273, 51-75

4. Finnveden S., Fraggstedt M., 2008, Waveguide finite elements for curved structures, Journal of Sound and Vibration, 312, 644-671

5. French Standard, 2007, Document: FA Rules: Prediction method by calculating the fire behavior of steel structures (in French), V2 CD-DTU-150, 92-702

6. Gavric L., 1995, Computation of propagative waves in free rail using a finite element technique, Journal of Sound and Vibration, 185, 420-432

7. Hayashi T., Song W.J., Rose J.L., 2003, Guided wave dispersion curves for a bar with an arbitrary cross-section, a rod and rail example, Ultrasonics, 41, 175-183

8. Jeyaraj P., Ganesan N., Padmanabhan C., 2009, Vibration and acoustic response of composite plate with inherent material damping in a thermal environment, Journal of Sound and Vibration, 320, 322-338

9. Kadoli R., Ganesan N., 2006, Buckling and free vibration analysis of functionally graded cylindrical shells subjected to temperature-specified boundary condition, Journal of Sound and Vibration, 289, 450-480

10. Kodur V., Kand S., Khalia W., 2012, Effect of temperature on thermal and mechanical properties of steel bolts, Journal of Materials in Civil Engineering, 24, 6, 765-774

11. Konstantinidis G., Drinkwater B.W., Wilcox P.D., 2006, The temperature stability of guided wave structural health monitoring systems, Smart Materials and Structures, 15, 967-976

12. Li H.Y., Hu J.D., Li J., Chen G., 2013, Effect of tempering temperature on microstructure and mechanical properties of AISI 6150 steel, Journal of Central South University, 20, 4, 866-870 